\title{
Revealed preferences with plural motives: axiomatic foundations of normative assessments in non-utilitarian welfare economics
}

\author{
Sebastian Silva-Leander ${ }^{1,2}$. Suman Seth ${ }^{1,3}$
}

(C) The Author(s) 2017. This article is published with open access at Springerlink.com

\begin{abstract}
This paper explores the possibility of defining a non-utilitarian normative standard for assessments of welfare and deprivation. The paper formalises a key aspect of Amartya Sen's critique of the assumption of consistent utility-maximisation in the revealed preference theory and proposes a generalisation of the standard Samuelsonian choice model for the case in which choices are based on plural motives (here, selfinterested and moral motives). Based on a set of intuitive assumptions about the way in which unobservable motives are linked to observable choices, we then construct an alternative normative ranking rule that can be used in non-utilitarian welfare economics to rank social outcomes or provide a normative basis for the construction of composite indices, for instance.
\end{abstract}

\section{Introduction}

Samuelsonian revealed preference theory (Samuelson 1938, 1948) provides the foundation for most contemporary economic analysis, positive and normative. Samuelson's theory finds conditions on observed choices that are consistent with individual preference maximization. However, recent work in behavioural economics (see, for instance,

Sebastian Silva-Leander

sebastian_silvaleander@yahoo.com

Suman Seth

S.Seth@leeds.ac.uk

1 Oxford Poverty and Human Development Initiative, Department of International Development, University of Oxford, Oxford, UK

2 Oxford Policy Management, 52 Cornmarket St, Oxford OX1 3HJ, UK

3 Economics Division, Leeds University Business School, University of Leeds, Maurice Keyworth Building, Leeds LS2 9JT, UK 
Rubinstein and Salant 2012; Camerer et al. 2005) and evidence from neuroscience (Harrison 2008) have challenged the notion that individuals maximize a single welldefined preference in making choices. Sen has criticised the model from a normative point of view for arbitrarily attaching value to individuals' actual choices, whatever those may be, without regard for whether those choices are the product of an informed rational deliberation process, or whether they have been conditioned by ignorance, oppressive social structures, adaptation or even addiction (Sen 1977, 1980, 1997, 2004).

This paper seeks to contribute to the growing body of literature in economics that looks at the possibility and implications of reformulating Samuelson's revealed preference theory to respond to the above mentioned criticism (see, for instance, Kalai et al. 2002; Green and Hojman 2007). The model operates at two levels. First, choices are made at the individual level through an internal decision-making mechanism based on underlying (unobservable) preference orderings, called motives. Individual choices are then combined into a social or collective choice through a collective (observable) decision making mechanism. Following Chambers and Hayashi (2012), we will adopt the viewpoint of an external scientific observer or economist, who attempts to impute underlying binary preference relations (motives) from observed individual or collective choices over pairs of goods using a standard Bayesian approach (see also Noor 2011, 2013). Advances in neurosciences may one day enable us to obtain direct evidence on motivational sources (see, for instance, Bernheim and Rangel 2009). However, this paper will be concerned with the question of how to assign normative value to observed choices in the absence of such evidence. The main result of the paper, captured in Proposition 2, is to show that it is possible to generate a normative ranking of social outcomes that is based on a non-utilitarian description of human nature and thus on a non-standard theory of choice.

The specific normative stance adopted in this paper follows Sen in considering that some motivations are more "legitimate" than others (e.g. rational deliberation vs. addiction), making it normatively relevant to aquire information, not only about observed choices, but also about underlying motives. ${ }^{1}$ The proposed model is nonstandard in that non-utilitarian motives are taken into account, but the methodology we adopt for analysing those preferences is standard in that the observable is a choice correspondence, or collection of choice correspondences.

In Sect. 2, we will introduce the notation and explain the overall structure of the model. In Sect. 3, we present the model and introduce the axioms that will be used to derive the results. The standard (Samuelsonian) model is nested in our model, when we exclude reasoned or moral motives. In Sect. 4, we present our main results. Section 5 concludes.

\footnotetext{
1 In so doing we thus depart from the interpretation of the revealed preference theory as a methodological statement (Gul and Pesendorfer 2005) and as a mere formalisation of the concept of preference as choice (Debreu 1954). For a critique of this interpretation and a justification of the chosen approach, we refer the reader to the writings of Sen (1977), Hirschman (1984) and Etzioni (1986).
} 


\section{Structure and notation}

The model works backwards from an external observer making inferences about the underlying motives that have led individuals or groups of individuals to choose particular options. The external observer then makes a normative ranking of the chosen options based on his/her positive assessment of the likely motives underpinning observed choices.

The notation used in this paper will be complicated by the fact that we use different symbols to describe the same object, depending on whether we are describing it from the point of view of the chooser or from the point of view of the external observer. This is deemed necessary to keep a clear distinction between normative, descriptive and inferencial statements.

Furthermore, the model comprises two levels of choice, namely an individual level and a collective level, where individual choices are combined into social choices. Here, we will use different subscripts to distinguish these different levels, namely lower case subscripts for individual choices, and upper case subscripts for collective choices.

\subsection{Chooser}

Let $N$ be the set of all possible individuals and let $X$ be the finite set of all possible options. We denote any non-empty subset of $X$ by $S$ and the set of all such non-empty subsets by $K$. If $S$ is the set of options available to an individual $i \in N$, then let $c_{i}(S) \subseteq S$ denote the choice set of $i$ or the set of chosen options from $S$ by $i$. For example, suppose $S=\{x, y\}$ for any $x, y \in X$ and $c_{i}(S)=\{x\}$. This means that individual $i$ has chosen option $x$ from the available options $x$ and $y$. Thus, $c_{i}(S)$ is the observable primitive of the model. In this paper, we treat $S$ as a variable within $K$ as in Sen (1971).

The observable choice set $c_{i}(S)$ has been generated from unobservable motives, $R_{i}$, through an internal decision making mechanism that is described below. Our model however does not impose any functional form that represents these motives. The decision maker $i$ 's motives are represented by a complete, transitive, and reflexive binary relation over any pairs of options, $x, y \in X$, where $x R_{i} y$ should be interpreted as meaning that " $x$ is at least as good as $y$ according to the motive $R_{i}$." The asymmetric and symmetric elements of $R_{i}$ will be designated, respectively, by $P_{i}^{*}$ and $I_{i}$. For the moment, we are not making any assumptions about how $R_{i}$ relates to $c_{i}(S)$, and are simply defining $R_{i}$ as a description of an individual's mental ranking of available options, prior to making the choice.

At the collective level, a social choice may be obtained by combining individual choices through a collective decision-making mechanism. For the sake of simplicity, we assume that each individual in $N$ has only one choice set, $c_{i}(S)$, generated from exactly two motives, $R_{i}^{U}$ and $R_{i}^{M}$, which will be described below. Our approach in this paper however could easily be generalised to more than two motives. Let $\mathcal{I} \subseteq N$ be any non-empty subset (group) of individuals. Note that we allow a group to consist of only one individual. Let us denote the set of all such subsets of individuals by $Q$. For any $\mathcal{I} \in Q$ that has reached consensus on a particular collective decision, we 
denote the collective choice set corresponding to that group simply as the intersection of individual observable choice sets: $c_{\mathcal{I}}(S) \equiv \cap_{i \in \mathcal{I}} c_{i}(S)$, where the number of people reaching consensus to a collective decision within group $\mathcal{I}$ is denoted by its cardinality $|\mathcal{I}|$. We assume $c_{\mathcal{I}}(S)$ is non-empty.

Suppose the collective choice of group $\mathcal{L} \in Q$ prevails as the social choice, which, in our framework, is treated as a concensus decision. We do not need to make any assumption about the way in which any consensus is reached, other than the fact that each individual $i \in \mathcal{L}$ has equal influence over the consensus decision. Our formulation can thus accommodate all possible decision making mechanisms, ranging from absolute dictatorship when the decisive group consists of only one individual, to unanimity rule when the consensus size is equal to the number of individuals in the population, to absolute majority rule when the concensus size is more than half of the number of individuals in the population.

For our purpose in the paper, we will make a distinction between two fundamental types of motives described by Harsanyi (1955) and Sen (1977). One is subjective, selfcentred or idiosyncratic motives and the other is ethical, moral or reasoned motives. The idiosyncratic motives are defined as yielding a private preference ordering $R_{i}^{U}$ $\forall i \in N$, such that there exists $i, j \in N, i \neq j$, such that $R_{i}^{U} \neq R_{j}^{U}$. Thus, idiosyncratic motives are defined by the fact that there exists at least two individuals for which the motives diverge. The reasoned motives, on the other hand, are defined as a singleton $R_{\mathcal{I}}^{M}$ for any group $\mathcal{I} \subseteq N$ such that $R_{i}^{M}=R_{\mathcal{I}}^{M} \forall i \in \mathcal{I}$. Reasoned motives are defined by the fact that they concord (i.e. are identical) across individuals within a group. Reasoned motives refer to the capacity of individual members of a group to recognise that there is a common group interest that transcends their individual interests. ${ }^{2}$ Note that each individual assesses options according to both motives, and the order of his or her assessments does not affect the individual's decision outcome. Moreover, these motives are assumed to be fixed and independent across individuals, which is reflected by a property that we refer to as independence in Sect. 3.1.

\subsection{External observer}

Because $R_{i}^{\text {' }}$ 's here represent mental states or motives, rather than actual choices, they are never directly observed, and will have to be inferred by the external observer from the outcome of a choice process. The external observer does this by applying a standard Bayesian deductive logic to available information, where observed choices are considered as the outcome of a stochastic process generated by unobservable events (underlying motives), which have a given probability of occurring. From the point of

\footnotetext{
2 The specific form of rationality invoked under $R_{\mathcal{I}}^{M}$ can be thought of as a weak form of Kantian "pure reason". Formally, Kant defines pure reason as the "faculty of the unity of the rules of understanding under principles" (Kant 1781, p. A302/B359), that is the capacity for abstraction or universalisation. Judgment, then is the capacity "of thinking the particular as contained under the universal" (Kant 1790, p. 5:179), that is to link a particular situation to a universal rule. In our model, $R_{\mathcal{I}}^{M}$ will coincide with a universal rule only in the specific case where $\mathcal{I}=N$ (see Proposition 1 below). Note that this definition of rationality does not require individuals to agree on all moral issues, since (a) $R_{\mathcal{I}}^{M}$ may only cover a small subset of options, and (b) final choices may be influenced also by idiosyncratic motives.
} 
view of the external observer who is making tentative inferences about unobservable phenomena, the underlying motives will thus be thought of as events.

For the simplicity of exposition, from this point forward we consider only a fixed pair of options $x, y \in X$ such that $x \neq y$ and introduce some additional notation. We use the notation $\Psi_{i}=\left\{\psi_{i} ; \chi_{i}\right\}$ to designate the set of possible events for chooser $i$ over the pair of options $x, y \in X$, where $\psi_{i}^{*}=y P_{i}^{*} x$ and $\chi_{i}^{*}=x P_{i}^{*} y .^{3}$ As usual, $P_{i}$. can be decomposed into weak preference relations, such that $\psi_{i}^{*} \equiv y R_{i} x \& \neg x R_{i} y$ and $\chi_{i} \equiv x R_{i} y \& \neg y R_{i} x$. Note that the external observer does not observe $\Psi_{i}$ or its elements as this decision making process is internal to chooser $i$.

The set of possible observable outcomes associated with $\Psi_{i}$ is written as $\Omega_{i}=$ $\left\{\omega_{i}^{\varnothing} ; \omega_{i}^{x} ; \omega_{i}^{y}\right\}$, where the elements in the set are defined as follows for the pair $x, y \in X$ and for any $i \in N$ :

- $\omega_{i}^{\varnothing} \equiv x, y \notin c_{i}(S)$ and $x, y \in S$ (i.e. $i$ chooses neither $x$ nor $y$ when both are available).

- $\omega_{i}^{x} \equiv x \in c_{i}(S)$ and $y \in S \backslash c_{i}(S)$ (i.e. $i$ chooses only $x$ when both $x$ and $y$ are available).

- $\omega_{i}^{y} \equiv y \in c_{i}(S)$ and $x \in S \backslash c_{i}(S)$ (i.e. $i$ chooses only $y$ when both $x$ and $y$ are available).

The role of the external observer will be to attempt to estimate the conditional probability $p\left(\Psi_{i} \mid \Omega_{i}\right)$, given the external observer's assumptions about the decision making process linking unobservable events $\Psi_{i}$ to observable outcomes $\Omega_{i}$.

As in the previous subsection, the subscript $\mathcal{I}$ will be used to describe the components pertaining to any group $\mathcal{I} \in Q$. Thus, $\chi_{\mathcal{I}}^{U}$, for instance, describes the unobserved event that $x P_{i}^{U} y$ occurs simultaneously for all $i \in \mathcal{I}$, i.e., $\chi_{\mathcal{I}}^{U} \equiv \cap_{i \in \mathcal{I}} \chi_{i}^{U}$. In other words, it describes a case in which there is a concordance of interests within the group $\mathcal{I}$. Similar group notation applies to other elements in the set of possible events. Similarly, $\omega_{\mathcal{I}}^{x}$ denotes the observable collective choice outcome corresponding to the consensus set $c_{\mathcal{I}}(S)$, generated by the collective decision making mechanism of group $\mathcal{I}$, whereby group $\mathcal{I}$ collectively chooses $x$ over $y$, when $y$ is available, i.e., $\omega_{\mathcal{I}}^{x} \equiv \cap_{i \in \mathcal{I}} \omega_{i}^{x}$. Similar group notation applies to other elements in the set of observable outcomes. The set of all possible finite collective decision-making outcomes observed by the external observer, $\omega_{\mathcal{I}}^{*}$, reached by all $\mathcal{I} \in Q$ is denoted by $Z$ (this includes individual choice outcomes), which is the domain of the ranking of social outcomes.

The end goal is to obtain a normative assessment of social outcomes. To this end, the external observer has to assign normative value to his inferred beliefs regarding the motives of the chooser(s). From this point of view, the outcomes of the choice process will be thought of as social states. Here, we will use the notation $\succcurlyeq$ to mean that a given social state "is at least as good as". Hence, $\omega_{\mathcal{I}}^{x} \succcurlyeq \omega_{\mathcal{I}}^{y}$ indicates that social state $x$ of group $\mathcal{I}$ is preferrable from a normative point of view to social state $y$ of group $\mathcal{I}$. Note that $\succcurlyeq$ has a symmetric and asymmetric element, denoted by $\sim$ and $\succ$, respectively. The normative ranking of social states described by $\succcurlyeq$ should not be

\footnotetext{
3 For simplicity, we leave out the case of indifference between options. In this paper, we are mainly concerned with collective choices typically taking the form of an exclusive choice between two or more options, for instance, in an election.
} 
confused with the positive description of individuals' actual preferences over those states, noted $R_{i}^{U}$ and $R_{i}^{M}$.

Note that $\succcurlyeq$ is only transitive on $Z$ and cannot necessarily be reduced to a transitive ranking on outcomes because it takes both the outcome and the group of individuals who comprise consensus on that outcome as inputs. For example, if groups $\mathcal{I}, \mathcal{J} \in Q$ both agree on the public provision of health care, we could have $\omega_{\mathcal{I}}^{x} \succ \omega_{\mathcal{J}}^{x}$ if $|\mathcal{I}|>|\mathcal{J}|$, which simply means that the normative force of group $\mathcal{I}$ 's preference for health care is greater than that of group $\mathcal{J}$ 's preferences for health care.

\section{Model}

In order to draw inferences about unobservable motives from observable choices, the external observer defines a system of conditional probabilities, $p(\cdot)$, which defines the set of possible outcomes that can be generated by a given combinations of events (motives). These constitute the observer's beliefs about the way in which motives generate observed choices. For tractability, we will impose some regularity assumptions (or rules for inference) on the set of conditional probabilities.

\subsection{Linking motives to social outcomes}

In the presence of dual preferences, the decision-making process will need to consist of at least three separate stages. In the first two stages, the options are assessed separately according to the two motives, $R_{i}^{U}$ and $R_{i}^{M}$. The specific order of the idiosyncratic and reasoned assessments does not influence the results of this paper. What matters is that two separate and independent assessments are carried out before the final choice is made, which is stage three.

Given that the external observer can only see the final choices made but not the underlying motives that have led to the observed choice, the observer needs to impose a set of plausible assumptions, which we refer to as properties, about the decisionmaking process linking underlying preferences to choice. The properties will be stated as a system of conditional probabilities characterising the decision-making process from the point of view of the external observer.

Anonymity (ANO) For all $i, j \in N$ such that $i \neq j$ and for $x, y \in X, p\left(\chi_{i}^{U} \mid \omega_{i}^{x}\right)=$ $p\left(\chi_{j}^{U} \mid \omega_{j}^{x}\right)$ and $p\left(\psi_{i}^{U} \mid \omega_{i}^{y}\right)=p\left(\psi_{j}^{U} \mid \omega_{j}^{y}\right)$.

The first property (ANO) captures the external observer's assumption that the probability that a given act is motivated by idiosyncratic motives is equal across all individuals.

Independence (IND) For any $\mathcal{I} \in Q$ such that $|\mathcal{I}|>1$ and for $x, y \in X, p\left(\chi_{\mathcal{I}}^{U}\right.$ | $\left.\omega_{\mathcal{I}}^{x}\right)=\prod_{i \in \mathcal{I}} p\left(\chi_{i}^{U} \mid \omega_{i}^{x}\right)$ and $p\left(\psi_{\mathcal{I}}^{U} \mid \omega_{\mathcal{I}}^{y}\right)=\prod_{i \in \mathcal{I}} p\left(\psi_{i}^{U} \mid \omega_{i}^{y}\right)$.

According the second property (IND), the external observer assumes that idiosyncratic motives of individuals are jointly independent within any subgroup of size greater than or equal to 2 . In other words, the external observer assumes that individual $i$ 's preference for, say, $x$ over $y$ is not influenced by whether other individuals within 
the same group prefer $x$ over $y$. This does not necessarily imply that the chooser's idiosyncratic motives must be independent of others' idiosyncratic motives, but simply that the external observer does not have any information on whether and how such relations exist. Inferring dependence in individual motives by the external observer will require further assumptions that we do not consider in this paper.

Completeness (COM) For any $\mathcal{I} \in Q$ and for $x, y \in X, p\left(\chi_{\mathcal{I}}^{U} \cup \chi_{\mathcal{I}}^{M} \mid \omega_{\mathcal{I}}^{*}\right)+p\left(\psi_{\mathcal{I}}^{U} \cup\right.$ $\left.\psi_{\mathcal{I}}^{M} \mid \omega_{\mathcal{I}}^{*}\right)=1$

The third property $(\mathrm{COM})$ states that a given observed act, $\omega_{\mathcal{I}}^{\cdot}$, must have been motivated either by $\chi_{\mathcal{I}}^{U} \cup \chi_{\mathcal{I}}^{M}$ or by $\psi_{\mathcal{I}}^{U} \cup \psi_{\mathcal{I}}^{M}$. Note that $\omega_{\mathcal{I}}^{\cdot}$ may represent either $\omega_{i}^{x}$ or $\omega_{i}^{y}$ or $\omega_{i}^{\varnothing}$.

Coherence $(\mathbf{C O H})$ For any $\mathcal{I} \in Q$ and for $x, y \in X, p\left(\psi_{\dot{\mathcal{I}}} \mid \omega_{\mathcal{I}}^{x}\right)=0$ and $p\left(\chi_{\dot{\mathcal{I}}} \mid\right.$ $\left.\omega_{\mathcal{I}}^{y}\right)=0$.

This fourth property $(\mathrm{COH})$ states that if a group $\mathcal{I}$ (which may consist of a single individual) is observed to have chosen $x$ over $y$, then the external observer infers that $\mathcal{I}$ could not have strongly preferred $y$ over $x$, neither under idiosyncratic motives nor under moral motives. Similar statement applies when $y$ is observed to be chosen over $x$. This is a Pareto axiom, stating that if preferences go in one direction, choice cannot go in the other direction. ${ }^{4}$

Uncertainty (UNC) For any $\mathcal{I}, \mathcal{J} \in Q \backslash\{N\}$ and for $x, y \in X, p\left(\chi_{\mathcal{I}}^{U} \cap \chi_{\mathcal{I}}^{M} \mid \omega_{\mathcal{I}}^{x}\right)=$ $p\left(\chi_{\mathcal{J}}^{U} \cap \chi_{\mathcal{J}}^{M} \mid \omega_{\mathcal{J}}^{x}\right)$ and $p\left(\psi_{\mathcal{I}}^{U} \cap \psi_{\mathcal{I}}^{M} \mid \omega_{\mathcal{I}}^{y}\right)=p\left(\psi_{\mathcal{J}}^{U} \cap \psi_{\mathcal{J}}^{M} \mid \omega_{\mathcal{J}}^{y}\right)$.

The final property (UNC) states that when the external observer observes that a group has chosen, say, $x$ over $y$, and it is possible for all idiosyncratic motives within that group to concord, the external observer acknowledges that there is a possibility of the decision being reached by a combination of both idiosyncratic and moral motives. However, in the absence of any information about the underlying decision making mechanisms, he assumes that the overlap between idiosyncratic and moral motives is the same across groups. ${ }^{5}$ This property is only used to obtain the result in Corollary 1.

The five properties impose natural restrictions on beliefs based on observed choices, which will help the external observer to draw inference on unobservable motives from observed choice outcomes. In order to assign normative value to these choices, we must introduce an additional axiom that links the positive inference to a normative rule. If, like Sen (1977), we consider that value stems from reason rather than from other preferences, it is logical to make the normative value of a given choice directly proportional on the probability that it is caused by $R_{i}^{M}$ and inversely proportional to the probability that it is caused by $R_{i}^{U}$. We do this by using the following normative

\footnotetext{
4 Note that in the single preference case, property $\mathrm{COH}$ reduces to $p\left(\psi_{i}^{U} \mid \omega_{i}^{x}\right)=0$, which could arguably be considered as a formal representation of Sen's $(1971,1977)$ interpretation of the Axiom of Revealed Preference (ARP) as a normative inference claim ("revelation") about underlying utility-maximising preferences (i.e. "if we observe that $i$ chooses $x$ over $y$, when $y$ is available, then we can infer that $i$ did not prefer $y$ to $x$ under the relation $R_{i}^{U}$ "). This should be contrasted against the usual interpretation of the ARP as a descriptive equivalence claim (i.e. "if $i$ chooses $x$ over $y$, when $y$ is available, we say that $i$ does not prefer $y$ to $x$ under the relation $R_{i}^{\prime \prime \prime) . ~}$

5 One may extend this simplified property by assuming a distribution of the probabilities of overlap.
} 
axiom, called Probability-Based Rankings, which states that the external observer (1) normatively prefers the social outcome that is less likely to have been caused by idiosyncratic motives (i.e. a convergence of self-interests), and (2) is normatively indifferent between two social states that have equal probability of having been caused by idiosyncratic motives. In other words, from the perspective of the external observer, a greater concordance of choices made under idiosyncratic motives would not carry more normative strength, as such concordance would be merely coincidental, and could be reversed if the external interests leading to the choice were to change.

Probability-Based Rankings (PBR) For $x, y \in X$ and for any $\mathcal{I}, \mathcal{J} \in Q$ and $\omega_{\mathcal{I}}^{x}, \omega_{\mathcal{J}}^{x} \in Z$

$$
\begin{aligned}
& \omega_{\mathcal{I}}^{x} \succ \omega_{\mathcal{J}}^{x} \Leftrightarrow p\left(\chi_{\mathcal{I}}^{U} \mid \omega_{\mathcal{I}}^{x}\right)<p\left(\chi_{\mathcal{J}}^{U} \mid \omega_{\mathcal{J}}^{x}\right) \\
& \omega_{\mathcal{I}}^{x} \sim \omega_{\mathcal{J}}^{x} \Leftrightarrow p\left(\chi_{\mathcal{I}}^{U} \mid \omega_{\mathcal{I}}^{x}\right)=p\left(\chi_{\mathcal{J}}^{U} \mid \omega_{\mathcal{J}}^{x}\right) .
\end{aligned}
$$

\section{Results}

In this section, we present our results in the form of Lemma 1, Propositions 1 and 2.

\subsection{Inference from collective choices}

The first result is captured in Lemma 1, which states that the probability that a group's choice is based on idiosyncratic motives is lower than for any of its proper subsets. The intuition behind Lemma 1 is that the more thorough the vetting process (i.e. the more individuals who have to consent to a decision), the less likely it is that the collective choice outcome will result from a mere coincidence of self-interests.

Lemma 1 For all $\mathcal{I}, A \in Q \backslash\{N\}$ and for $x, y \in X$, we can use properties $A N O$ and IND to infer that: $A \subset \mathcal{I} \Rightarrow p\left(\chi_{\mathcal{I}}^{U} \mid \omega_{\mathcal{I}}^{x}\right) \leq p\left(\chi_{A}^{U} \mid \omega_{A}^{x}\right)$.

Proof Let $\mathcal{I}, A \in Q \backslash\{N\}$ be two non-empty groups of individuals, such that $A \subset \mathcal{I}$. It then follows that $0<|A|<|\mathcal{I}|$.

By property ANO, we already know that for all $i, j \in N$ such that $i \neq j$,

$$
p\left(\chi_{i}^{U} \mid \omega_{i}^{x}\right)=p\left(\chi_{j}^{U} \mid \omega_{j}^{x}\right)
$$

By property IND along with Eq. (2), we get

$$
p\left(\chi_{\mathcal{I}}^{U} \mid \omega_{\mathcal{I}}^{x}\right)=\left(p\left(\chi_{i}^{U} \mid \omega_{i}^{x}\right)\right)^{|\mathcal{I}|}
$$

and

$$
p\left(\chi_{A}^{U} \mid \omega_{A}^{x}\right)=\left(p\left(\chi_{i}^{U} \mid \omega_{i}^{x}\right)\right)^{|A|} .
$$

Given that $|A|<|\mathcal{I}|$, then comparing Eqs. (3) and (4), we obtain $p\left(\chi_{\mathcal{I}}^{U} \mid \omega_{\mathcal{I}}^{x}\right) \leq$ $p\left(\chi_{A}^{U} \mid \omega_{A}^{x}\right)$. 
Lemma 1 has an intuitive corollary that may be obtained by using the UNC property along with properties $\mathrm{COM}$ and $\mathrm{COH}$.

Corollary 1 For all $\mathcal{I}, A \in Q \backslash\{N\}$ and for $x, y \in X$, we can use properties ANO, IND, COM, COH, and UNC to infer that: $A \subset \mathcal{I} \Rightarrow p\left(\chi_{\mathcal{I}}^{M} \mid \omega_{\mathcal{I}}^{x}\right) \geq p\left(\chi_{A}^{M} \mid \omega_{A}^{x}\right)$.

Proof We already know from Lemma 1 that by properties ANO and IND,

$$
p\left(\chi_{\mathcal{I}}^{U} \mid \omega_{\mathcal{I}}^{x}\right) \leq p\left(\chi_{A}^{U} \mid \omega_{A}^{x}\right)
$$

Then, by property $\mathrm{COH}$, we know that for any $\mathcal{I} \in Q$ and for $x, y \in X, p\left(\psi_{\mathcal{I}}^{U}\right.$ | $\left.\omega_{\mathcal{I}}^{x}\right)=0$ and $p\left(\psi_{\mathcal{I}}^{M} \mid \omega_{\mathcal{I}}^{x}\right)=0$ and thus, $p\left(\psi_{\mathcal{I}}^{U} \cup \psi_{\mathcal{I}}^{M} \mid \omega_{\mathcal{I}}^{x}\right)=0$. Combined with property COM leads to, for any $\mathcal{I} \in Q$ and for $x, y \in X$ :

$$
p\left(\chi_{\mathcal{I}}^{U} \cup \chi_{\mathcal{I}}^{M} \mid \omega_{\mathcal{I}}^{x}\right)=1 .
$$

It then follows that,

$$
p\left(\chi_{\mathcal{I}}^{U} \cup \chi_{\mathcal{I}}^{M} \mid \omega_{\mathcal{I}}^{x}\right)=p\left(\chi_{A}^{U} \cup \chi_{A}^{M} \mid \omega_{A}^{x}\right) .
$$

Expanding both sides of Eq. (7), we obtain,

$$
\begin{aligned}
p\left(\chi_{\mathcal{I}}^{U} \mid \omega_{\mathcal{I}}^{x}\right)+p\left(\chi_{\mathcal{I}}^{M} \mid \omega_{\mathcal{I}}^{x}\right)-p\left(\chi_{\mathcal{I}}^{U} \cap \chi_{\mathcal{I}}^{M} \mid \omega_{\mathcal{I}}^{x}\right)= & p\left(\chi_{A}^{U} \mid \omega_{A}^{x}\right)+p\left(\chi_{A}^{M} \mid \omega_{A}^{x}\right) \\
& -p\left(\chi_{A}^{U} \cap \chi_{A}^{M} \mid \omega_{A}^{x}\right) .
\end{aligned}
$$

Finally, by property UNC, we know that

$$
p\left(\chi_{\mathcal{I}}^{U} \cap \chi_{\mathcal{I}}^{M} \mid \omega_{\mathcal{I}}^{x}\right)=p\left(\chi_{A}^{U} \cap \chi_{A}^{M} \mid \omega_{A}^{x}\right) .
$$

Hence, by combining Eqs. (5), (8) and (9), we obtain $p\left(\chi_{\mathcal{I}}^{M} \mid \omega_{\mathcal{I}}^{x}\right) \geq p\left(\chi_{A}^{M} \mid \omega_{A}^{x}\right)$.

Under the external observer's assumption, stated in property UNC, Corollary 1 intuitively states that more thorough the vetting process, the more likely it is that the collective choice outcome will result from moral motives.

The following proposition follows from the definition of idiosyncratic and reasoned motives. Indeed, in the limit, as $\mathcal{I}$ tends to $N$, we can think of a hypothetical consensus involving all possible individuals in $N$ with all possible combinations of motives.

Proposition 1 For $x, y \in X$, if $\mathcal{I}=N$, then we can use the definitions of idiosyncratic and reasoned motives along with properties COM and COH to infer that: $p\left(\chi_{\mathcal{I}}^{U} \cup \chi_{\mathcal{I}}^{M}\right.$ । $\left.\omega_{\mathcal{I}}^{x}\right)=p\left(\chi_{\mathcal{I}}^{M} \mid \omega_{\mathcal{I}}^{x}\right)=1$.

Proof By the general property of additivity of probabilities, we have:

$$
p\left(\chi_{\mathcal{I}}^{U} \cup \chi_{\mathcal{I}}^{M} \mid \omega_{\mathcal{I}}^{x}\right)=p\left(\chi_{\mathcal{I}}^{U} \mid \omega_{\mathcal{I}}^{x}\right)+p\left(\chi_{\mathcal{I}}^{M} \mid \omega_{\mathcal{I}}^{x}\right)-p\left(\chi_{\mathcal{I}}^{U} \cap \chi_{\mathcal{I}}^{M} \mid \omega_{\mathcal{I}}^{x}\right) .
$$


By the construction of $\chi_{\mathcal{I}}^{U}$, we can re-write Eq. (10) as:

$$
p\left(\chi_{\mathcal{I}}^{U} \cup \chi_{\mathcal{I}}^{M} \mid \omega_{\mathcal{I}}^{x}\right)=p\left(\cap_{i \in \mathcal{I}} \chi_{i}^{U} \mid \omega_{\mathcal{I}}^{x}\right)+p\left(\chi_{\mathcal{I}}^{M} \mid \omega_{\mathcal{I}}^{x}\right)-p\left(\cap_{i \in \mathcal{I}} \chi_{i}^{U} \cap \chi_{\mathcal{I}}^{M} \mid \omega_{\mathcal{I}}^{x}\right)
$$

Given that $\mathcal{I}=N$, by the definition of idiosyncratic motives, we know that $\cap_{i \in N} \chi_{i}^{U}=\varnothing$. Hence, $p\left(\cap_{i \in \mathcal{I}} \chi_{i}^{U} \mid \omega_{\mathcal{I}}^{x}\right)=p(\varnothing)=0$.

Also, $p\left(\cap_{i \in N} \chi_{i}^{U} \cap \chi_{\mathcal{I}}^{M} \mid \omega_{\mathcal{I}}^{x}\right)=p\left(\varnothing \cap \chi_{\mathcal{I}}^{M} \mid \omega_{\mathcal{I}}^{x}\right)=p(\varnothing)=0$.

Consequently, Eq. (11) boils down to:

$$
p\left(\chi_{\mathcal{I}}^{U} \cup \chi_{\mathcal{I}}^{M} \mid \omega_{\mathcal{I}}^{x}\right)=p\left(\chi_{\mathcal{I}}^{M} \mid \omega_{\mathcal{I}}^{x}\right)
$$

By properties $\mathrm{COH}$ and COM, finally, we have $p\left(\chi_{\mathcal{I}}^{U} \cup \chi_{\mathcal{I}}^{M} \mid \omega_{\mathcal{I}}^{x}\right)=1$ as in (6). Hence, $p\left(\chi_{\mathcal{I}}^{M} \mid \omega_{\mathcal{I}}^{x}\right)=1$.

Proposition 1 makes the point that we can only say with certainty that an observable outcome arises from moral motives in the hypothetical case in which there is a consensus amongst all possible individuals in $N$ who have ever existed and could ever exist. In all other real-life cases, we will thus be dealing with uncertain and tentative normative judgements.

\subsection{Normative ranking of social choices}

We now prove Proposition 2, which states that, given the properties and the PBR axiom outlined in Sect. 3, the normative strength of an option will be directly dependent on the number of individuals who are able to agree on its value.

Proposition 2 For all $\mathcal{I}, \mathcal{J} \in Q \backslash\{N\}$, for $x, y \in X$ and for a system of conditional probabilities $p(\cdot)$ that satisfies properties ANO and IND, if the induced relation $\succcurlyeq$ satisfies $P B R$ and is binary, transitive and reflexive on observed choices $\omega_{\mathcal{I}}^{x}, \omega_{\mathcal{J}}^{x} \in Z$, then $|\mathcal{I}|>|\mathcal{J}| \Rightarrow \omega_{\mathcal{I}}^{x} \succcurlyeq \omega_{\mathcal{J}}^{x}$.

Proof For any $\mathcal{I}, \mathcal{J} \in Q \backslash\{N\}$, suppose $|\mathcal{I}|>|\mathcal{J}|$ and let $A \subset \mathcal{I}$ such that $|A|=|\mathcal{J}|$. As $p(\cdot)$ satisfies properties ANO and IND, by Lemma 1 , we already know that

$$
A \subset \mathcal{I} \Rightarrow p\left(\chi_{\mathcal{I}}^{U} \mid \omega_{\mathcal{I}}^{x}\right) \leq p\left(\chi_{A}^{U} \mid \omega_{A}^{x}\right)
$$

Following PBR then we get $p\left(\chi_{\mathcal{I}}^{U} \mid \omega_{\mathcal{I}}^{x}\right) \leq p\left(\chi_{A}^{U} \mid \omega_{A}^{x}\right) \Rightarrow \omega_{\mathcal{I}}^{x} \succcurlyeq \omega_{A}^{x}$.

If we can show that $p\left(\chi_{A}^{U} \mid \omega_{A}^{x}\right)=p\left(\chi_{\mathcal{J}}^{U} \mid \omega_{\mathcal{J}}^{x}\right)$, then that will complete our proof. By property ANO, we already know that for all $i, j \in N$ such that $i \neq j$,

$$
p\left(\chi_{i}^{U} \mid \omega_{i}^{x}\right)=p\left(\chi_{j}^{U} \mid \omega_{j}^{x}\right)
$$

Given that $A, \mathcal{J} \subseteq N$, the relation in Eq. (14) holds for all $i, j \in A$ as well as for all $i, j \in \mathcal{J}$. 
Note that we have assumed both $|A|>1$ and $|\mathcal{J}|>1$. Then by property IND, we obtain

$$
p\left(\chi_{\mathcal{J}}^{U} \mid \omega_{\mathcal{J}}^{x}\right)=\prod_{i \in \mathcal{J}} p\left(\chi_{i}^{U} \mid \omega_{i}^{x}\right)
$$

and

$$
p\left(\chi_{A}^{U} \mid \omega_{A}^{x}\right)=\prod_{i \in A} p\left(\chi_{i}^{U} \mid \omega_{i}^{x}\right) .
$$

By our assumption of $|A|=|\mathcal{J}|$, combining Eqs. (14), (15) and (16) we obtain,

$$
p\left(\chi_{A}^{U} \mid \omega_{A}^{x}\right)=p\left(\chi_{\mathcal{J}}^{U} \mid \omega_{\mathcal{J}}^{x}\right)
$$

Then, by PBR (1b), we get $p\left(\chi_{A}^{U} \mid \omega_{A}^{x}\right)=p\left(\chi_{\mathcal{J}}^{U} \mid \omega_{\mathcal{J}}^{x}\right) \Rightarrow \omega_{A}^{x} \sim \omega_{\mathcal{J}}^{x}$.

Finally, since $\succcurlyeq$ is transitive over $Z$, it follows that $\omega_{\mathcal{I}}^{x} \succcurlyeq \omega_{A}^{x}$ and $\omega_{A}^{x} \sim \omega_{\mathcal{J}}^{x} \Rightarrow$ $\omega_{\mathcal{I}}^{x} \succcurlyeq \omega_{\mathcal{J}}^{x}$.

Proposition 2 captures Sen's (2004) notion of normative validation through a process of inter-rational testing, e.g. through public discussion or reasoned argument (see also Popper 1980, p. 111). As such, it supports the intuitively appealing idea that majority consensus carries more "normative" force when the majority forming the consensus is larger (e.g. the United States vs. Lichtenstein, or the Universal Declaration of Human Rights, vs. the European Convention on Human Rights). At the same time, it recognises the limitations of such processes, highlighting the historically verified fact that free democratic decisions do not by themselves guarantee outcomes (except in the hypothetical case of a consensus involving an infinite number of preference profiles following the result presented in Proposition 1). ${ }^{6}$

\section{Conclusions}

In a recent paper, Ravallion (2011) rightly criticised the weights used in multidimensional indices for lacking the sort of theoretical justification that allows neoclassical economists to claim that "market prices are defensible weights on quantities in measuring national income" (Ravallion 2011, p. 3). However, as Sen (1987) has argued repeatedly, the normative claims of neoclassical economics are contingent on the acceptance of an essentially utilitarian normative standpoint and description of human nature, which is far from uncontroversial. Furthermore, even if we accept such premises, there are well-studied theoretical and normative shortcoming in the classical model of the rational self-interested decision-maker (Sen 1977; Rubinstein and Salant 2012, etc.).

\footnotetext{
6 One alternative interpretation of the results in this paper could be as follows: suppose an agent has a true preference that is fixed across time but in every period there is an idiosyncratic shock (modelled as giving rise to a new binary relation) that lead to choices that are "errors" with regards to the agent's true preference. The data constitutes this agent's choices in different time periods. The result then states that the longer the stretch of time where the agent chooses the same alternative from a fixed menu, the more likely it is to reveal her true preferences.
} 
In this paper, we have tried to explore the possibility of relaxing some of the most enduring assumptions of this model in order to make it more compatible with the nonutilitarian description of human nature proposed by Sen. In particular, we have shown that it is possible to develop a normative ranking that starts from the assumption of plural motivations. The model focuses on the specific issue of non-utilitarian normative rankings, and does not look into other criticisms that have been levied against the standard choice model, regarding, for instance, incoherent choices (Hammond 1976), temptation (Kopylov 2012), regret (Stoye 2011), etc. As such, it can be subjected to many of the same critiques and variations as the standard model to explore real-world deviations from this theoretical benchmark.

We believe that the findings presented in this paper have practical implications for normative economic assessments, notably when using so-called multidimensional poverty indices, since it provides a theoretical foundation that is currently lacking for assigning weights in the construction of such indices. In particular, the model presented in this paper is compatible with a rights-based approach to determination of weights, based, for instance, on normative frameworks, such as the international declaration of human rights or other documents that have gone through a process of inter-rational validation. Potentially, the findings of this paper, could also have applications in other areas of normative economics, which currently rely, explicitly or implicitly, on the axiom of revealed preference to assign normative value to social outcomes.

Acknowledgements We wish to thank Gaston Yalonetsky, Constanze Binder, Sabina Alkire, and Frances Stewart for helpful comments on earlier drafts of this paper. We also wish to thank the two anonymous referees and the journal editor for their exceptionally thorough and constructive comments, as well as their patience. All mistakes remain our own.

Open Access This article is distributed under the terms of the Creative Commons Attribution 4.0 International License (http://creativecommons.org/licenses/by/4.0/), which permits unrestricted use, distribution, and reproduction in any medium, provided you give appropriate credit to the original author(s) and the source, provide a link to the Creative Commons license, and indicate if changes were made.

\section{References}

Bernheim DB, Rangel A (2009) Beyond revealed preference: choice theoretic foundations for behavioral welfare economics. Q J Econ 124(1):51-104

Camerer C, Loewenstein G, Prelec D (2005) Neuroeconomics: how neuroscience can inform economics. J Econ Lit 43(1):9-64

Chambers CP, Hayashi T (2012) Choice and individual welfare. J Econ Theory 147(5):1818-1849

Debreu G (1954) Representation of a preference ordering by a numerical function. Decis Process 3:159-165

Etzioni A (1986) The case for a multiple-utility conception. Econ Philos 2:159-183

Green J, Hojman D (2007) Choice, rationality and welfare measurement. Discussion paper number 2144, Harvard Institute of Economic Research, Harvard University, Cambridge, Massachusetts

Gul F, Pesendorfer W (2005) The case for mindless economics. In: Caplin A, Schotter A (eds) The foundations of positive and normative economics: a handbook. Oxford University Press, Oxford

Hammond PJ (1976) Changing tastes and coherent dynamic choice. Rev Econ Stud 43:159-173

Harrison GW (2008) Neuroeconomics: a critical reconsideration. Econ Philos 24(3):303-344

Harsanyi JC (1955) Cardinal welfare, individualistic ethics, and interpersonal comparisons of utility. J Polit Econ 63:309-321

Hirschman AO (1984) Against parsimony: three easy ways of complicating some categories of economic discourse. Bull Am Acad Arts Sci 37(8):11-28 
Kalai G, Rubinstein A, Spiegler R (2002) Rationalising choice functions by multiple rationales. Econometrica 70(6):2481-2488

Kant I (1781) Critique of pure reason (kritik der reinen vernunft). In: Gruyte WD, Holger EG (eds) Akademieausgabe von Immanuel Kants Gesammelten Werken (1969 ed.), vol IV. Royal German Academy of Sciences, Berlin, pp 1-238

Kant I (1790) Critique of judgment, (kritik der urtheilskraft). In: Gruyter WD (ed) Kants gesammelte Schriften (1902 ed.), vol 5. Royal German Academy of Sciences, Berlin

Kopylov I (2012) Perfectionism and choice. Econometrica 80:1819-1843

Noor J (2011) Temptation and revealed preference. Econometrica 79(2):601-644

Noor J (2013) Removed preferences. J Econ Theory 148(4):1463-1486

Popper K (1980) The logic of scientific discovery. Hutchinson, London

Ravallion M (2011) Mashup indices of development. World Bank Res Obs 27:1-32

Rubinstein A, Salant Y (2012) Eliciting welfare preferences from behavioural data sets. Rev Econ Stud 79(1):375-387

Samuelson PA (1938) A note on the pure theory of consumer's behaviour. Economica 5(17):61-71

Samuelson PA (1948) Consumption theory in terms of revealed preference. Economica 15(60):243-253

Sen AK (1971) Choice functions and revealed preference. Rev Econ Stud 38(3):307-317

Sen AK (1977) Rational fools: a critique of the behavioural foundations of economic theory. Philos Public Aff 6:317-344

Sen AK (1980) Equality of what? The tanner lectures on human values 1. Stanford University, Stanford

Sen AK (1987) On ethics and economics. Basil Blackwell, Oxford

Sen AK (1997) Choice, welfare and measurement. Harvard University Press, Cambridge

Sen AK (2004) Rationality and freedom. Harvard University Press, Cambridge

Stoye J (2011) Axioms for minimax regret choice correspondences. J Econ Theory 146:2226-2251

Sebastian Silva-Leander holds a D.Phil. in Economics from the University of Oxford. His main research interests include social choice, poverty and inequality measurement and the operationalisation of the capability approach. He has also published several papers and participated in conferences on issues related to horizontal inequality and conflict.

Suman Seth is a lecturer in Economics at the University of Leeds and a research associate at the Oxford Poverty and Human Development Initiative. He has served as a consultant to the United Nations Development Programme, the World Bank, and the Asian Development Bank. His primary interest lies in the area of Development Economics with special emphasis on measurement methodologies and policy-oriented applications of multidimensional welfare and poverty measures. He has published two co-authored books on poverty measurement. 